\title{
Intracerebral Hemorrhage in a Patient with Multiple Sclerosis Receiving Interferon Beta- $1 \alpha$
}

\author{
Sareh Shahmohammadi, ${ }^{1}$ Seyed Ehsan Mohammadianinejad, ${ }^{1}$ Mohammad Ali Sahraian, ${ }^{1}$ and
}

\author{
Abdorreza Naser Moghadasi ${ }^{1,}$ \\ ${ }^{1}$ MS Research Center, Neuroscience Institute, Tehran University of Medical Sciences, Tehran, Iran \\ "Corresponding author: Abdorreza Naser Moghadasi, MS Research Center, Neuroscience Institute, Tehran University of Medical Sciences, Tehran, Iran. Tel: +98-2166348571, Fax: \\ +98-2166348570, E-mail: abdorrezamoghadasi@gmail.com
}

Received 2016 October 08; Accepted 2016 December 05.

\begin{abstract}
Interferon beta-1 $\alpha$ (IFN $\beta-1 \alpha$ ) is one of the most widely used disease-modifying drugs, resulting in reduced annualized relapse rates and MRI activity in patients with multiple sclerosis (MS). Years of experience has indicated the relative safety of this class of medications in patients with MS. Despite its overall safety profile, an increasing number of reports have revealed serious adverse events in form of angiopathic complications in the preceding years. Herein, we present the case of a patient with chronic MS under treatment with IFN $\beta$-1 $\alpha$, presenting with intracerebral hemorrhage (ICH) without any other identifiable causes. The present report, along with various cases of bleeding in patients receiving IFN, indicates a possible association between IFN therapy and ICH.
\end{abstract}

Keywords: Intracerebral Hemorrhage, Multiple Sclerosis, Interferon Beta-1 $\alpha$

\section{Introduction}

Intracerebral hemorrhage (ICH) is the third most common cause of stroke, following embolic and atherothrombotic occlusion (1). While the most common cause of primary ICH is hypertension, secondary causes such as vascular malformation, aneurysm, coagulopathy, and substance- or drug-induced factors should be always considered in the absence of a history of hypertension and presence of underlying medical and/or neurological disorders (2).

Multiple sclerosis (MS) is one of the most common chronic and debilitating neurological disorders in young and middle-aged individuals, with major impacts on the patient's quality of life (3). The probability of superimposed medical and neurological disorders is always a concern in these patients (4). Although the correlation between hemorrhagic events and MS has not been reported per se, there are different reports of hemorrhagic events in the presence of interferon (IFN) therapy in the literature.

Herein, we present the case of an MS patient, who was receiving IFN therapy for a long period of time and was referred to our hospital with ICH.

\section{Case Presentation}

A 52-year-old woman with secondary progressive MS was referred to the emergency department with reduced consciousness. Her symptoms had started the night before admission with an acute headache, which was described as generalized and throbbing; the patient had no prior experience of such headaches. Drowsiness, without nausea or vomiting, was reported upon admission. Vital signs including blood pressure (BP) were reported to be normal during a home visit by the emergency medical service provider.

Upon admission to the emergency department, the patient's BP was $130 / 80 \mathrm{mmHg}$. Neurological examination showed drowsiness, associated with left upper limb weakness. She had a nine-year history of MS, with secondary progression over the past four years; the patient was wheelchair-bound for two and a half years. Treatment with IFN $\beta-1 \alpha$ ( $44 \mathrm{mcg}$, three times per week) was started since the onset of the disease and continued up to hospital admission, regardless of the progressive course of the disease. Prior history of trauma or cardiovascular risk factors, such as hypertension, hyperlipidemia, smoking, diabetes, and associated systemic disorders, was not reported in the patient. She was receiving fluoxetine, tizanidine, clonazepam, and oxybutynin for symptomatic treatment.

Laboratory tests including routine blood and coagulation studies were normal (Table1). Brain computed tomography (CT) scan revealed massive ICH in the right hemisphere, involving the basal ganglia with an extension to temporal and parietal lobes (Figure 1A). Repeated CT scan after four days showed the expansion of hematoma and a smaller focus of hematoma in the left basal ganglia (Figure 1B). The brain magnetic resonance imaging (MRI) scan did not reveal any vascular malformation, aneurysm, or brain tumor (Figure 1C and 1D). Neurosurgical intervention was not performed due to the increased risk-to-benefit ratio. 
Table 1. Including Routine Blood and Coagulation Studie

\begin{tabular}{lc}
\hline Variable & Value \\
\hline WBC & 5900 \\
\hline Hb & 16.5 \\
\hline HCT & 44.7 \\
\hline PLT & 172000 \\
\hline BS & 107 \\
\hline Urea & 23 \\
\hline Cr & 0.74 \\
\hline PT & 13.5 \\
\hline PTT & 29.9 \\
\hline INR & 1.01 \\
\hline
\end{tabular}
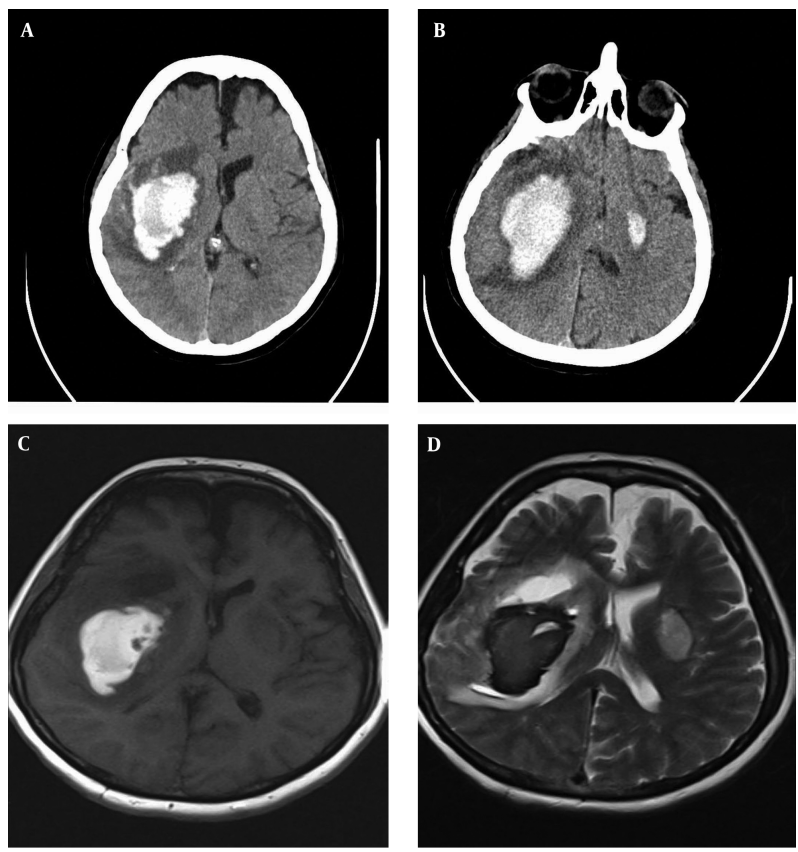

Figure 1. A, massive ICH in the right hemisphere involving the basal ganglia with an extension to temporal and parietal lobes; B, repeated CT scan after four days indicated the expansion of hematoma and a smaller focus of hematoma in the left basal ganglia; C and D, the brain MRI did not reveal vascular malformation, aneurysm, or brain tumor.

\section{Discussion}

IFN $\beta$ - $1 \alpha$ has been one of the most widely used treatment agents in MS patients over the past years. This is mainly due to the overall safety profile of this drug, which has been confirmed by its long-term use since its introduction in the mid 1990's $(5,6)$. More common side-effects, such as local injection reactions, flu-like symptoms, hepa- totoxicity of variable degrees, and hematological and thyroid abnormalities are well-known and more easily manageable $(7,8)$.

While an almost good safety profile is considered for IFN- $\beta$, an increasing number of reports have indicated some previously unknown adverse events, which may be potentially serious in some cases. Among these, thrombotic microangiopathy, arterial hypertension, Raynaud's phenomenon, livedo reticularis, capillary leak syndrome, pulmonary artery hypertension, and retinopathy warrant consideration (9-16). There seems to be a common angiopathic mechanism involved in these more recent complications. The effect of IFN- $\alpha$ with a similar chemical structure on vascular events has been described in the literature before (17).

Some of the adverse events have occurred with the long-term use of high-dose, high-frequency IFN therapy, suggesting a dose-dependent manner. However, some events occur soon after the initiation of IFN therapy. Therefore, according to evidence available in the literature, it seems that we should be more vigilant with regard to the continuation of IFN therapy in a subgroup of patients with MS, who are less likely to benefit from this type of therapy.

Adverse vascular events mentioned above may be associated with microangiopathy and capillary leak syndrome. This in fact can be a good reason to suppose a correlation between IFN- $\beta$ and vascular changes. The present report is in line with several previous cases of $\mathrm{ICH}$, associated with IFN therapy. Some reports have indicated this complication in some patients with hepatitis C, treated with IFN- $\alpha$ alone or in combination with antiviral therapy (18-20).

Hemorrhagic and ischemic retinopathy, subdural hematoma, vaginal bleeding, and intraocular hemorrhage have been also reported in patients receiving IFN therapy for different conditions (e.g., hepatitis) of variable durations; some of these complications resolved after drug discontinuation (21-24). Our patient was a middleaged woman who had a long-term history of MS with a secondary progressive course for three years, receiving IFN $\beta$ - $1 \alpha$ therapy. Currently, there is no evidence indicating the benefits of IFN treatment in MS patients with a progressive course without superimposed relapses.

\subsection{Conclusion}

The increasing number of reports, revealing more serious but uncommon adverse effects of IFN therapy in patients with MS, highlights the need for more attention to this treatment. Angiopathic complications, including ischemic and hemorrhagic events, may be due to IFN therapy if alternative causes are excluded. According to the available evidence, we should be more vigilant with regard to the continuation of IFN (as an apparently safe drug) 
therapy in a subgroup of MS patients who may not benefit from this type of treatment.

\section{Footnote}

Financial Disclosure: The authors declare no financial interests related to the materials of this study.

\section{References}

1. Ikram MA, Wieberdink RG, Koudstaal PJ. International epidemiology of intracerebral hemorrhage. Curr Atheroscler Rep. 2012;14(4):300-6. doi: 10.1007/s11883-012-0252-1. [PubMed: 22538431].

2. Badjatia N, Rosand J. Intracerebral hemorrhage. Neurologist. 2005;11(6):311-24. [PubMed: 16286875].

3. Lublin FD. The incomplete nature of multiple sclerosis relapse resolution. J Neurol Sci. 2007;256 Suppl 1:S14-8. doi: 10.1016/j.jns.2007.01.062. [PubMed: 17337274].

4. Marrie RA, Reider N, Cohen J, Stuve O, Sorensen PS, Cutter G, et al. A systematic review of the incidence and prevalence of autoimmune disease in multiple sclerosis. Mult Scler. 2015;21(3):282-93. doi: 10.1177/1352458514564490. [PubMed: 25533299].

5. Sanford M, Lyseng-Williamson KA. Subcutaneous recombinant interferon-beta-1a $(\operatorname{Rebif}(\mathrm{R}))$ : a review of its use in the treatment of relapsing multiple sclerosis. Drugs. 2011;71(14):1865-91. doi: 10.2165/11207540-000000000-00000. [PubMed: 21942977].

6. Hartung HP, Haas J, Meergans M, Tracik F, Ortler S. [Interferon-beta1b in multiple sclerosis therapy: more than 20 years clinical experience]. Nervenarzt. 2013;84(6):679-704. doi: 10.1007/s00115-013-3781-0. [PubMed: 23669866].

7. Galetta SL, Markowitz C. US FDA-approved disease-modifying treatments for multiple sclerosis: review of adverse effect profiles. CNS Drugs. 2005;19(3):239-52. [PubMed:15740178].

8. Deghima S, Chentli F. [Interferons and thyroid abnormalities: literature review]. Rev Med Liege. 2012;67(3):147-51. [PubMed: 22611832].

9. Cavoli GL, Passantino R, Tortorici C, Bono L, Ferrantelli A, Rotolo U. Comment on thrombotic microangiopathy induced by interferonbeta therapy. Clin Nephrol. 2012;78(6):506-7. [PubMed: 23164420].

10. Vosoughi R, Marriott JJ. Thrombotic microangiopathy in Interferon Beta treated multiple sclerosis patients: Review of literature and report of two new cases. Mult Scler Relat Disord. 2014;3(3):321-5. doi 10.1016/j.msard.2013.12.007. [PubMed: 25876469].

11. Modrego PJ, Gazulla J. Arterial hypertension induced by interferon beta $1 \mathrm{~b}$ in a patient with multiple sclerosis. Mult Scler. 2012;18(11):16556. doi: 10.1177/1352458512441272. [PubMed: 22419671].
12. Rot $U$, Ledinek AH. Interferons beta have vasoconstrictive and procoagulant effects: a woman who developed livedo reticularis and Raynaud phenomenon in association with interferon beta treatment for multiple sclerosis. Clin Neurol Neurosurg. 2013;115 Suppl 1:S79-81. doi: 10.1016/j.clineuro.2013.09.027. [PubMed: 24321162].

13. Rodriguez-Vico JS, Mendoza A, Duarte J. [Livedo reticularis following subcutaneous injection of interferon beta-1b]. Rev Neurol. 2010;51(1):62. [PubMed: 20568071].

14. Niederwieser $G$. Lethal capillary leak syndrome after a single administration of interferon beta-1b. Neurology. 2000;54(7):1545-6. [PubMed: 10751284].

15. Gibbons E, Promislow S, Davies RA, Chandy G, Stewart DJ, Vladamir $\mathrm{CD}$, et al. Reversible pulmonary arterial hypertension associated with interferon-beta treatment for multiple sclerosis. Can Respir J. 2015;22(5):263-5. [PubMed: 26083539].

16. Gaetani L, Menduno PS, Cometa F, Di Gregorio M, Sarchielli P, Cagini $\mathrm{C}$, et al. Retinopathy during interferon-beta treatment for multiple sclerosis: case report and review of the literature. $J$ Neurol. 2016;263(3):422-7. doi: 10.1007/s00415-015-7879-0. [PubMed: 26292794].

17. Al-Zahrani H, Gupta V, Minden MD, Messner HA, Lipton JH. Vascular events associated with alpha interferon therapy. Leuk Lymphoma. 2003;44(3):471-5. doi: 10.1080/1042819021000055066. [PubMed: 12688317].

18. Niederwieser G, Bonelli RM, Kammerhuber F, Reisecker F, Koltringer P. Intracerebral haemorrhage under interferon-beta therapy. Eur J Neurol. 2001;8(4):363-4. [PubMed: 11422436].

19. Nishiofuku M, Tsujimoto T, Matsumura Y, Toyohara M, Yoshiji H, Yamao J, et al. Intracerebral hemorrhage in a patient receiving combination therapy of pegylated interferon alfa-2b and ribavirin for chronic hepatitis C. Internal Med. 2006;45(7):483-4. doi:10.2169/internalmedicine.45.1685.

20. Ferencz S, Batey R. Intracerebral haemorrhage and hepatitis $C$ treatment. JViral Hepat. 2003;10(5):401-3. [PubMed: 12969193].

21. Okuno H, Hirota T, Shiozaki Y, Inoue K, Hagihara M, Sugasawa K, et al. [Interferon-associated retinopathy]. Nihon Rinsho. 1994;52(7):1919-23. [PubMed: 7521439].

22. Goto T, Ohshima S, Miura K, Shibuya T, Sato W, Dohmen T, et al. A chronic subdural hematoma in a patient receiving combination therapy with pegylated interferon alfa-2b and ribavirin for chronic hepatitis C. Inter Med. 2013;52(18):2057-60. doi: 10.2169/internalmedicine.52.0791.

23. Pakulski LA, DiMarco LM. Severe vaginal bleeding associated with recombinant interferon beta-1B. Ann Pharmacother. 1997;31(1):50-2. [PubMed: 8997465].

24. Kuga K, Hasumura S, Nagamori S, Toda G, Kitahara K. Intraocular hemorrhage developing during interferon therapy. Internal Med. 1996;35(1):15-8. doi: 10.2169/internalmedicine.35.15. 